NSF-KITP-03-81, DAMTP-2003-90, McGill-03/21, SU-ITP-03/26

\title{
de Sitter String Vacua from Supersymmetric D-terms
}

\author{
C.P. Burgess, ${ }^{1}$ R. Kallosh ${ }^{2}$ and F. Quevedo ${ }^{3}$ \\ ${ }^{1}$ Physics Department McGill University \\ 3600 University Street \\ Montréal, Quebec, Canada, H3A $2 T 8$. \\ ${ }^{2}$ Department of Physics, Stanford University, \\ Stanford, CA 94305-4060, USA. \\ ${ }^{3}$ DAMTP, Centre for Mathematical Sciences \\ University of Cambridge, \\ Cambridge CB3 0WA UK.
}

\begin{abstract}
We propose a new mechanism for obtaining de Sitter vacua in type IIB string theory compactified on (orientifolded) Calabi-Yau manifolds similar to those recently studied by Kachru, Kallosh, Linde and Trivedi (KKLT). dS vacuum appears in KKLT model after uplifting an AdS vacuum by adding an anti-D3-brane, which explicitly breaks supersymmetry. We accomplish the same goal by adding fluxes of gauge fields within the D7-branes, which induce a D-term potential in the effective $4 \mathrm{D}$ action. In this way we obtain $\mathrm{dS}$ space as a spontaneously broken vacuum from a purely supersymmetric $4 \mathrm{D}$ action. We argue that our approach can be directly extended to heterotic string vacua, with the dilaton potential obtained from a combination of gaugino condensation and the D-terms generated by anomalous $U(1)$ gauge groups.
\end{abstract}




\section{Contents}

1. Introduction 1

2. Modulus Stabilization 2

3. de Sitter Vacua 5

4. Heterotic String Vacua 9

5. Conclusions 11

6. Acknowledgements 12

\section{Introduction}

Recently Kachru, Kallosh, Linde and Trivedi (KKLT) [1] found the first explicit realization of $4 \mathrm{D}$ de Sitter space as a solution to the low-energy equations of string theory. This is a significant achievement given the importance that de Sitter space has acquired from the recent data on the acceleration of the universe and also for its close relation with the inflationary scenario.

The proposal of KKLT combines several mechanisms that lift the vacuum degeneracy of supersymmetric string models. First they introduce background fluxes for NS and RR forms to fix all of the complex-structure moduli of a Calabi-Yau compactification [2, 3, 4, 5, 6]. Second, they focus on models having only one Kähler modulus, and fix this remaining modulus using nonperturbative effects combined with the remnant superpotential produced by the fluxes, typically leading to a supersymmetric 4D anti-de Sitter vacuum. Finally, the addition of an anti-D3-brane provides an extra source of positive potential energy which depends on the Kähler modulus, and lifts the minimum to a $4 \mathrm{D}$ de Sitter vacuum.

All steps except the last one can be understood within the context of an effective $4 \mathrm{D}$ supergravity, but since the addition of the anti-D3-brane breaks supersymmetry, 
the effective $4 \mathrm{D}$ theory cannot be put into the standard $4 \mathrm{D}$ supergravity form. ${ }^{1}$ This kind of explicit breaking of supersymmetry considerably complicates the analysis of the low-energy theory, because the loss of supersymmetry removes much of the theoretical control over the types of interactions which can be induced. This motivates searching for ways to obtain de Sitter space in string theory, but within a framework described by a fully supersymmetric action.

In this note we do so by modifying the last step of the KKLT stabilization process. Instead of adding an anti-brane, we turn on fluxes for yet another field: the gauge fields living inside the D7 branes. This extra degree of freedom is already contained within the original scenario and does not require adding sources such as anti-branes which are difficult to interpret as an F- or a D-term of a potential of a supersymmetric theory. Nevertheless, the addition of these fluxes has the similar effect of adding a positive term to the scalar potential, which can be interpreted as a D-term of the effective 4D supersymmetric action. It is the competition of this D-term potential with the superpotential contribution which breaks supersymmetry spontaneously and leads to a 4D de Sitter geometry.

Another method for replacing the antibrane in the KKLT construction is to obtain models whose potential at the no scale level admits a minimum with $D W \neq 0$ so that the potential has an additional term similar to the antibrane contribution to the potential [7].

\section{Modulus Stabilization}

Type IIB strings have RR and NS-NS antisymmetric 3-form field strengths, $H_{3}$ and $F_{3}$ respectively, which can wrap on 3 -cycles of the compactification manifold, leading to quantized background fluxes

$$
\frac{1}{4 \pi^{2} \alpha^{\prime}} \int_{A} F_{3}=M, \quad \frac{1}{4 \pi^{2} \alpha^{\prime}} \int_{B} H_{3}=-K
$$

where $K$ and $M$ are arbitrary integers and $A$ and $B$ label the different 3-cycles of the Calabi-Yau manifold.

Fluxes have proven to be very efficient at fixing many of the string moduli, including the axion-dilaton field of type IIB theory, $S=e^{\phi}+i a$ [2] 3]. A very general

\footnotetext{
${ }^{1}$ It may be possible to write the $4 \mathrm{D}$ action in a manifestly supersymmetric way if certain massive fields are 'integrated in' to act as the superpartners of fields appearing in the non-supersymmetric $4 \mathrm{D}$ effective action.
} 
analysis of orientifold models of type IIB, or its equivalent realization in terms of $F$-theory, has been done in [3]. In the $F$ theory approach, the geometrical picture corresponds to an elliptically fibered four-fold Calabi-Yau space $Z$ with base space $\mathcal{M}$ and the elliptic fiber corresponding to the axion-dilaton field $S$.

An important consistency condition coming from tadpole cancellation implies a relationship between the charges of D-branes, O-planes, and fluxes, which can be written as follows:

$$
N_{D 3}-N_{\bar{D} 3}+N_{f l u x}=\frac{\chi(Z)}{24} .
$$

Here the left-hand side counts the number of D3-branes and anti-branes, as well as the flux contribution to the RR charge:

$$
N_{\text {flux }}=\frac{1}{2 \kappa_{10}^{2} T_{3}} \int_{M} H_{3} \wedge F_{3} .
$$

The right-hand side of (2.2) refers to the Euler number of the four-fold manifold, $Z$, or in terms of orientifolds of type IIB, to the contribution of the D3-brane charge due to orientifold planes and D7-branes. Here $\kappa_{10}$ is the string scale in $10 \mathrm{D}$ and $T_{3}$ is the tension of the D3 branes.

From the point of view of the effective 4D theory, the fluxes generate a superpotential of the Gukov-Vafa-Witten form [ [由]:

$$
W=\int_{M} G_{3} \wedge \Omega,
$$

where $G_{3}=F_{3}-i S H_{3}$ with $S$ the dilaton field and $\Omega$ is the unique $(3,0)$ form of the corresponding Calabi-Yau space. An important feature of this superpotential is that it is independent of all of the compact space's Kähler moduli, so none of these moduli are fixed by these kinds of fluxes.

The simplest models have the fewest possible number of Kähler moduli: one. It defines the overall breathing mode of the underlying Calabi-Yau space. Denoting the 10D metric by $d s_{10}^{2}=L^{-6} d s_{4}^{2}+L^{2} d s_{6}^{2}$, this Kähler mode can be written $T=X+i Y$, where $X=L^{4} / g_{s}$ and $Y$ is an axion field coming from the RR 4-form. ( $T=i \rho$ in the conventions of [3, 国.) Since this modulus does not appear in $W$, it is not fixed by the fluxes, and the corresponding flat direction can be understood within the effective $4 \mathrm{D}$ theory because it takes the no-scale form [8]. That is, the low-energy Kähler potential is:

$$
K=\tilde{K}\left(\varphi_{i}, \varphi_{i}^{*}\right)-3 \log \left(T+T^{*}\right)
$$


with $\tilde{K}$ the Kähler potential for all of the other fields, $\varphi_{i}$, except for $T$. The corresponding F-term potential then takes the form

$$
\begin{aligned}
V_{F} & =e^{K}\left(K^{I \bar{J}} D_{I} W \overline{D_{J} W}-3|W|^{2}\right) \\
& =e^{K}\left(K^{i \bar{j}} D_{i} W \overline{D_{j} W}\right)
\end{aligned}
$$

where $i, j$ label the fields $\varphi^{i}$, while the labels $I, J$ include both the $\varphi^{i}$ and $T$. Here $K^{i \bar{j}}$ is the inverse of the Kähler metric, $K_{i \bar{j}}=\partial_{i} \partial_{\bar{j}} K$, and $D_{i} W=\partial_{i} W+W \partial_{i} K$ is the superpotential's Kähler covariant derivative. The second equality follows because the $T$-dependence of the Kähler potential is such that the contribution $K^{T \bar{T}}\left|D_{T} W\right|^{2}$ precisely cancels the term $-3|W|^{2}$. This is a special property of no-scale models [8]. Being positive definite, the global minimum of this potential lies at zero, with all fields except for $T$ fixed by the conditions $D_{i} W=0$. This minimum is supersymmetric if $D_{T} W=W=0$, and not supersymmetric otherwise.

In order to fix $T$ KKLT proceed as follows. First, they choose fluxes to obtain a vacuum in which supersymmetry is broken by the $T$ field, because $W=$ $W_{0} \neq 0$. Then they consider a nonperturbative superpotential, either generated by Euclidean D3-branes or by gaugino condensation within a non-abelian gauge sector of $N$ wrapped D7-branes. Since the gauge coupling for such a gauge group is $8 \pi^{2} / g_{Y M}^{2}=2 \pi L^{4} / g_{s}=2 \pi X$, well-established arguments imply a non-perturbative superpotential of the form $W_{n p}=A e^{-a T}$ [9], for appropriate constants $A$ and $a$.

Combining the two sources of superpotentials

$$
W=W_{0}+A e^{-a T},
$$

gives an effective scalar potential for the field $T=X+i Y$ of the form:

$$
V_{F}=\frac{1}{8 X^{3}}\left\{\frac{1}{3}\left|2 X W^{\prime}-3 W\right|^{2}-3|W|^{2}\right\},
$$

having a non-trivial minimum at finite $T$ as well as the standard runaway behaviour towards infinity. The non-trivial minimum corresponds to negative cosmological constant, and so gives rise to a supersymmetric AdS vacuum.

This is reminiscent of the standard situation in heterotic string theory regarding the potential for the dilaton field $S$ coming from the racetrack scenario, after having fixed the field T. More general superpotentials have been considered in [10], in which the superpotential takes the form:

$$
W=\sum_{i} A_{i} e^{-a_{i} T}
$$


where the sum can be finite or infinite. The finite case is the standard racetrack scenario [11]. The structure of the scalar potential is such that it has one or many anti-de Sitter minima, all of which are supersymmetric.

\section{3. de Sitter Vacua}

In order to obtain de Sitter vacua, KKLT add anti-D3 branes to the above construction, while still satisfying the tadpole condition (2.2). Semiclassically, this has the effect of adding an extra non-supersymmetric term (neither F- nor D-term) to the scalar potential of the form:

$$
V=V_{F}+\frac{k}{X^{2}}
$$

with the constant $k=2 a_{0}^{4} T_{3} / g_{s}^{4}$ parameterizing the lack of supersymmetry of the potential. Here $a_{0}$ is the warp factor at the location of the anti-D3 branes and $T_{3}$ is the anti-brane tension. The net effect of this addition to the potential is that, for suitable values of $k$, the original AdS minimum gets lifted to a dS one with broken supersymmetry. Fig. (11) gives a sample plot of the potential obtained in this way.

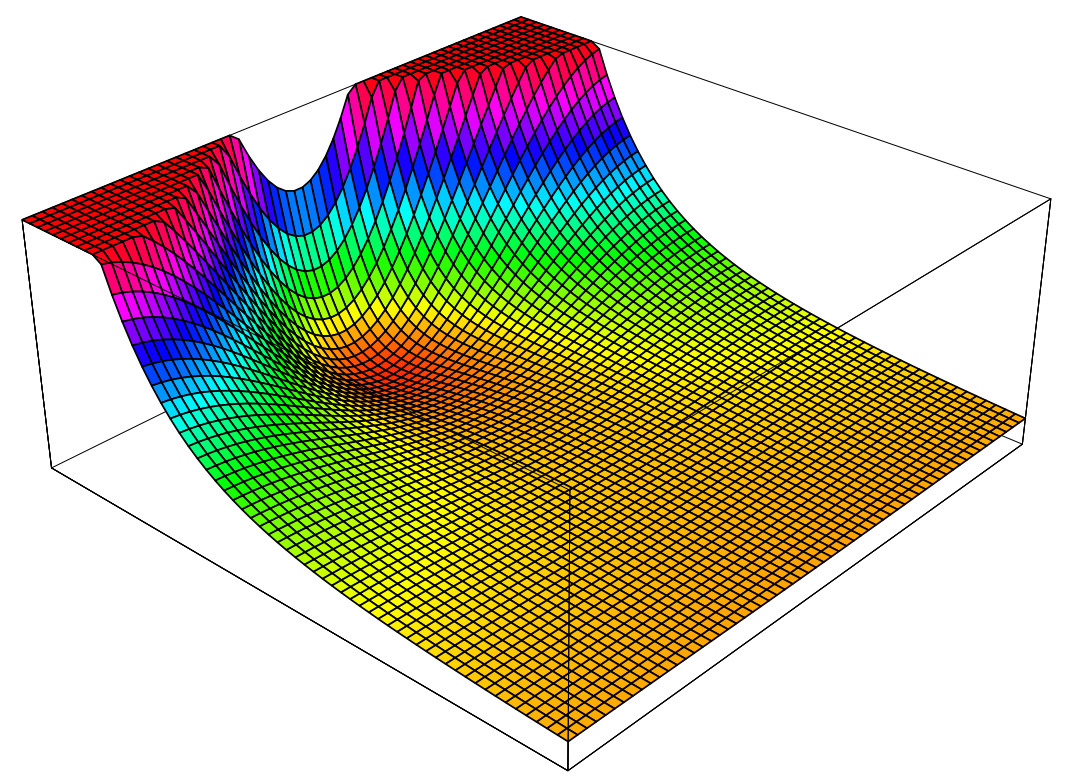

Figure 1: The scalar potential considered in [1] with a de Sitter minimum.

We will depart from the KKLT proposal and choose not to introduce anti-D3branes, and instead consider the possibility of turning on non-trivial fluxes for the gauge fields living on the D7 branes. From the linear D7 action, these fluxes will 
naturally induce a term in the $4 \mathrm{D}$ effective action of the form:

$$
T_{7} \int_{\Gamma} d^{4} y \sqrt{g_{8}} F^{m n} F_{m n}=\frac{2 \pi E^{2}}{X^{3}}
$$

where $\Gamma$ is the 4-cycle about which the D7 branes wrap, $T_{7}$ their tension, $E$ is a measure of the strength of the flux and the conventional factor of $2 \pi$ is for later convenience. The $X$ dependence can be inferred by a simple scaling analysis, using $\sqrt{g_{8}} g^{m n} g^{p q} \propto L^{-12}$.

The contribution of this term by itself is similar to the contribution induced by the introduction of anti-D3 branes. The only difference is the power of $X$ in the FI term. $^{2}$

The supersymmetry of this type of term from the $4 \mathrm{D}$ point of view has been studied in the past elsewhere, and was identified as arising as a field-dependent Fayet-Iliopoulos (FI) D-term in the $\mathcal{N}=1$ supersymmetric effective action [13, 14]. In general, type A branes - corresponding to D-branes wrapping 3-cycles - couple to complex-structure moduli as FI terms, while branes of type B - corresponding to D-branes wrapping around cycles of even dimensionality and with magnetic fluxes inside - couple to the Kähler-structure moduli as FI terms [13]. It is the second of these which is of interest for the present purposes. ${ }^{3}$

The full 4D-supersymmetric contribution to the scalar potential corresponding to eq. (3.2) therefore takes the form:

$$
V_{D}=\frac{g_{Y M}^{2}}{2} D^{2}=\frac{2 \pi}{X}\left(\frac{E}{X}+\sum q_{I}\left|Q_{I}\right|^{2}\right)^{2},
$$

where we again use $4 \pi / g_{Y M}^{2}=X$. The $Q_{I}$ represent any matter fields which are charged - with charges $q_{I}$ — under the $U(1)$ gauge group for which the fluxes provide a FI factor, such as can arise from D3-D7 strings. Eq. (3.3) assumes for simplicity a minimal Kähler potential for $Q_{I}$ of the form $\left|Q_{I}\right|^{2}$, although this is not crucial for the later discussion.

If the additional fields are minimized at $Q_{I}=0$, then the total potential

$$
V=V_{F}+V_{D}
$$

behaves very similarly to the KKLT potential, with $V_{D}=2 \pi E^{2} / X^{3}$ playing the role played by their anti-brane term. The net result for $X$ is therefore qualitatively the

\footnotetext{
${ }^{2}$ The power of $X$ in the D3-brane term was -3 in ref. [1], and this was corrected in ref. [12] to be -2 due to the appearance of an extra power of $X$ in the warp factor.

${ }^{3}$ We thank A. Uranga for a conversation on this point.
} 
same as for KKLT, but with the difference that we use only a fully supersymmetric 4D effective action. Whether the potential has de Sitter or anti-de Sitter minima depends on the values of the flux parameter $E$. If $E$ is too small, the minimum remains anti de Sitter, but if it belongs to a range of slightly larger values, then there is a de Sitter minimum. Beyond a particular critical value, the runaway behaviour of the pure FI potential dominates and the local minimum disappears, leaving only the runaway minimum at $X \rightarrow \infty$.

It is important for this argument that any fields $Q_{I}$ indeed get minimized at $Q_{I}=0$, since the stabilization of the de Sitter vacuum cannot occur along the lines described here if the $Q_{I}$ instead adjust to ensure $V_{D}=0$. Whether this happens for a particular string vacuum is model dependent, but we pause here to describe what can be said on reasonably general grounds.

If we would consider a system of D7 brane with fluxes and a D3 brane at some distance as in [15], we would have to take into account D3-D7 strings corresponding to positive and negative charged fields. They are stretched between separated D7 and D3 branes and their mass depends on the distance between branes. When D3 brane dissolves onto D7 brane with fluxes, the D-flatness condition $V_{D}=0$ is realized. Here, however, it is more appropriate to consider a single D7 brane with fluxes. If the D3-D7 separation is very large, then the D3-D7 open strings can be very massive, perhaps leading to no charged fields $Q_{I}$ in the effective $4 \mathrm{D}$ theory. A similar situation may occur if there are only fluxes but no D3 branes.

Note also that due to the presence of a flux on a D7 brane, a D5 brane charge may be generated, in general, via a Chern-Simons term $\int F_{2} \wedge C_{6}$. For our purpose we may consider either the situations where $\int F_{2}$ over a 2-cycle inside of a 4-cycle, which D7 brane wraps, vanishes, or a situation when somewhere far there is an antiD5-brane so that the total D5 charge vanishes. In orientifold models the orientifold action tends to change the sign of the gauge fields inside the D7 branes and then odd powers of $F_{2}$ do not contribute to tadpoles, in particular, D5 brane tadpoles are absent ${ }^{4}$. A D3 brane tadpole, generated by $\int C_{4} \wedge F_{2}^{2}$ only contributes to an extra term to equation (2.2) that can always be taken care of by the choice of the original flux contribution.

Second, it can happen that even if the $Q_{I}$ 's appear in the $4 \mathrm{D}$ effective theory, in some circumstances the scalar potential is minimized at $Q_{I}=0$. Even if $W$

\footnotetext{
${ }^{4}$ We thank R. Blumenhagen, S. Kachru and R. Rabadán for the useful conversations on these issues.
} 
is independent of $Q, V_{F}$ typically depends on $Q$ because the Kähler derivative is $\left|D_{I} W\right|^{2}=\left|K_{I} W\right|^{2}=\left|Q_{I} W\right|^{2}$. As is clear from these expressions, $V_{F}$ is often minimized by $Q_{I}=0$. Since the $Q$-dependent terms in $V_{F}$ are proportional to $X^{-3}$, while those in $V_{D}$ vary like $X^{-1}$, for large enough $X$ it is preferable for $Q_{I}$ to adjust to minimize $V_{D}$ rather than $V_{F}$. Conversely, for smaller $X$ the relative importance of $V_{F}$ for the minimization of $Q_{I}$ increases. An extreme case arises if only fields having a charge with the same sign as $E$ appear nontrivially in $W$, in which case there is no value for these fields for which $V_{D}=0 .{ }^{5}$

This last situation is analogous to the hybrid D-term inflation models [17]. Some of the superfields, which are left out of the superpotential, have positive charge only and therefore, for positive FI terms they have positive mass squared and vanish at the minimum of the potential ${ }^{6}$. The negatively charged fields which would be able to cancel the contribution from fluxes should be absent in this model since they would have a negative mass squared, unless they appear in the particular form (as in Dterm inflation models) in the superpotential. However, the original D-term inflation model [17] as well as the brane construction [15] realizing the D-term inflation in string theory, do not include the 4D space-dependent volume moduli and need to be properly generalized before the complete picture can be clarified. For our specific purpose of describing a possibility of a de Sitter minimum in presence of fluxes on D7 branes, the absence of negatively charged fields seems to be a sufficient condition for the absence of tachyons as well as for vanishing $Q_{I}$-fields at the minimum.

Although our starting point was the assumption of nonvanishing electromagnetic flux, our results only depend on the existence of a FI term, regardless of its origin. The only requirement is that the FI term depends on the (real part of the) $T$-field as an appropriately negative power of $X$. Let us pause here to understand this point better.

A sufficient condition for the existence of the FI term is the existence of a GreenSchwarz type of coupling of the form $B \wedge F$ between a $U(1)$ gauge field and the

\footnotetext{
${ }^{5}$ In general we do not need to have the matter fields to vanish. As long as the D-term part of the potential is non-zero at the minimum, it may do the job of lifting the vacuum to de Sitter space. In ref. [16], a particular example was worked out where it was shown that the combination of gaugino condensation and FI D-terms implies supersymmetry breaking in the sense that the D-term was not zero at the minimum.

${ }^{6}$ For the earlier work on single field inflation models with anomalous FI terms and a charged inflaton see [18].
} 
antisymmetric tensor, $B$, dual to the imaginary part of $T .^{7}$ This kind of coupling can arise, for instance, from the Chern-Simons coupling of the D7 brane, which takes the form:

$$
\int_{\mathcal{M}_{8}} C \wedge\left(\operatorname{Tr} e^{\frac{i F}{2 \pi}}\right) .
$$

Expanding the exponential produces the coupling $C_{\mu \nu m n} F_{\rho \sigma} F_{p q} \epsilon^{\mu \cdots q}$, where, as usual, Greek indices denote ordinary spacetime and Latin indices run over the Calabi-Yau dimensions. This precisely gives rise to the $B \wedge F$ term in the presence of nonvanishing background fluxes.

The necessity for a FI term follows from supersymmetry and gauge invariance, because a $B \wedge F$ term becomes $A_{\mu} \partial^{\mu} Y$ when expressed in terms of the axion, $Y$, which is dual to $B$. But gauge invariance then requires that $Y$ and $A_{\mu}$ must always appear together in the combination $\partial_{\mu} Y-q A_{\mu}$ for some constant $q$, and supersymmetry then implies that the Kähler potential can depend on $T$ only through the combination $T+T^{*}+c V^{8}$. Here $V$ is the gauge superfield including $A_{\mu}$ and $c$ is a constant (proportional to $E$ ). Expansion of the Kähler function, $K\left(T+T^{*}+c V\right)$, into components then induces a FI D-term proportional to $\partial K /\left.\partial V\right|_{V=0}$. Notice that whenever $K$ has the no-scale form, $K=-3 \ln \left(T+T^{*}+c V\right)$, the resulting auxiliary field $\mathrm{D}$ is proportional to $E / X$, as required by expression (3.3) for $V_{D}$ above, so that the potential has a standard form $\frac{1}{2} g^{2} D^{2}$.

Clearly our construction should generalize to any string vacuum for which such a $B \wedge F$ coupling appears. A broad (but not exhaustive) class of vacua for which it does consists of those for which there is an anomalous $U(1)$ in the low-energy theory, with $Y$ participating in the Green-Schwarz anomaly cancellation.

\section{Heterotic String Vacua}

Most of the past work on moduli potentials was done for heterotic string vacua, and potentials very similar to the ones appearing in the type IIB case were actually

\footnotetext{
${ }^{7}$ Recall that $Y$ is dual to the two-index field $B_{\mu \nu}$ defined by $C_{\mu \nu a b}=B_{\mu \nu} J_{a b}$ where $C_{M N P Q}$ is the original 4-index field of type IIB string theory and $J_{a b}$ is the Kähler form of the Calabi-Yau [3].

${ }^{8}$ Notice that, since $T$ transforms under the $U(1)$ interactions, a simple exponential of $T$ in the non-perturbative superpotential is not invariant under the corresponding $U(1)$ and needs to be compensated by the dependence of the superpotential on other fields. This, being model dependent, is beyond the scope of the present note. Nevertheless, it would be interesting to fully study a particular example with the matter field dependence under control.
} 
computed in that approach. A fixing of all string moduli has not yet been achieved for heterotic vacua, although work in this direction is now in progress by several groups [19]. In all cases when particular moduli were assumed fixed, the potentials for some of the remaining fields were sometimes calculable and were always found to be minimized for $4 \mathrm{D}$ anti-de Sitter vacua. One might wonder if there is a way to lift those vacua to the de Sitter case as well. At first sight, this seems difficult if we follow the KKLT scenario, since there is no analogue of the anti-D3-brane in the heterotic case. The same objection does not apply to the analysis as presented in the previous section, however, which is straightforward to generalize to the case of heterotic strings.

Nonperturbative superpotentials were studied in order to fix the "model independent' moduli fields, $T$ and $S$, of the heterotic string using the standard tree-level Kähler potential [20]:

$$
K=-\log \left(S+S^{*}\right)-3 \log \left(T+T^{*}\right) .
$$

Since $S$ is in this case the gauge coupling, the nonperturbative superpotential takes the 'racetrack' form:

$$
W(S, T)=\sum_{i} A_{i}(T) e^{-a_{i} S},
$$

where the $S$ dependence comes from gaugino condensation for different gauge groups, and the $T$ dependence can be obtained either from threshold corrections to the gauge coupling or by just requiring invariance under $T$-duality of the heterotic string [21, 22, 23]. We do not here consider any details regarding the functional dependence on $T$, but just content ourselves with pointing out that once $T$ is fixed we are left with a potential, $V_{F}$, for $S$ having the same form as the ones considered in the previous section for $T$. The generic potential of this form fixes the field $S$ at a supersymmetric or non-supersymmetric minimum with a negative (anti-de Sitter) cosmological constant.

It is also true that in generic heterotic models there is usually at least one anomalous $U(1)$ group. The anomaly is cancelled by a four-dimensional version of the Green-Schwarz mechanism, using the NS-NS antisymmetric field $B_{\mu \nu}$. Writing the dual of this field as $\partial_{\mu} a=\epsilon_{\mu \nu \rho \sigma} \partial^{\nu} B^{\rho \sigma}$, the couplings required by anomaly cancellation are $a F_{\mu \nu} \tilde{F}^{\mu \nu}$ and $B \wedge F$. Recall that $a$ is the axion field that makes up the imaginary part of the field $S$ and $F_{\mu \nu}$ is the field strength of the anomalous $U(1)$ gauge group. 
As is argued above (and is well known [24]), the existence of the $B \wedge F$ coupling implies (from gauge-invariance and supersymmetry) the existence of a FI term for the corresponding $U(1)$. We are again led to a D-term potential of the form:

$$
V_{D}=\frac{1}{S+S^{*}}\left(\frac{\operatorname{Tr} q}{48 \pi^{2}} \frac{1}{\left(S+S^{*}\right)^{2}}+\sum q_{I}\left|Q_{I}\right|^{2}\right)^{2},
$$

where the first term in the bracket is the FI term.

We see that the situation is completely analogous to our previous discussion for the type IIB string, with the roles of $S$ and $T$ interchanged. After considering the full scalar potential $V=V_{F}+V_{D}{ }^{9}$ we can have a local minimum with vanishing charged fields $Q_{I}$ and with $S$ fixed at a de Sitter minimum.

Whether a de Sitter minimum is really achieved is a model-dependent question. The important quantity to follow is the coefficient $\operatorname{Tr} q$. If there are many charged fields giving rise to a relatively large 'anomaly' Tr $q$, the D-term can dominate and lift the anti-de Sitter minimum to a de Sitter one. Otherwise the minimum remains anti-de Sitter. If $\operatorname{Tr} q$ is too large, then the FI term dominates the potential and may completely remove the minimum at finite $S$.

\section{Conclusions}

In this paper we generalize the KKLT construction [1] of de Sitter string theory vacua for which all of the moduli are fixed. The main difference between our approach and the previous one is the derivation from string theory of a metastable de Sitter space via a spontaneous supersymmetry breaking. It is achieved in the framework of the effective 4D supersymmetric effective action, through the interplay of F- and D-terms in the scalar potential. The virtue of keeping the low-energy supersymmetry manifest is the extra control this gives over the form of the low-energy Lagrangian, which is more difficult for the anti-D3-branes used by KKLT.

There are several issues in our proposal which may need to be examined in more detail for particular examples. Central among these is the question of finding systems for which the matter fields $Q_{I}$ do not cancel the contribution from fluxes to $V_{D}$. A second important issue is to understand whether new moduli arise when fluxes are turned on the D7 brane. Finally, it would be worth understanding in more

\footnotetext{
${ }^{9}$ For discussions on the combination of F-terms and D-terms induced by anomalous $U(1)$ 's see for instance [16].
} 
detail the relation of our flux proposal to the D3/D7 system of the type described in [15]. Notice that the D3/D7 system is analogous to Seiberg-Witten non-commutative theories 25] and the D0/D4 system of branes where the FI terms are related to fluxes on the D4 brane.

Even though our technique is described in a supersymmetric context, our result is very similar to the one obtained by KKLT by adding an anti-D3-brane. ${ }^{10}$

In contrast with the previous scenario, ours appears to immediately generalize to the heterotic string cases that have been studied in the past. It would be interesting to approach the possibility of obtaining D-brane inflation [26, 15] from our scenario, along the lines of [12].

We believe that our results set the existence of de Sitter space in string theory on firmer grounds, and are likely to be among the first of many new constructions of de Sitter vacua in string theory.

\section{Acknowledgements}

We thank the organizers of the KITP workshop on string cosmology for providing the perfect environment to start this work. We would like to thank P. Binetruy, J. Blanco-Pilado, R. Blumenhagen, R. Brustein, C. Escoda, M. Gómez-Reino, S. Kachru, S. Kovacs, P. Kumar, A. Linde, J. Maldacena, V.S. Nemani, R. Rabadán, E. Silverstein, H. Tye and A. Uranga for many discussions on these and related subjects. This research was supported in part by the NSF under grant No. PHY99-07949. C.B. is also funded by NSERC (Canada), FCAR (Québec) and McGill University. The work of R. K. is supported also by the NSF grant PHY-0244728. F.Q. is partially funded by PPARC.

\section{References}

[1] S. Kachru, R. Kallosh, A. Linde and S. P. Trivedi, "De Sitter vacua in string theory," Phys. Rev. D 68, 046005 (2003) [arXiv:hep-th/0301240].

[2] A. Strominger, "Superstrings With Torsion," Nucl. Phys. B 274 (1986) 253; S. Sethi, C. Vafa and E. Witten, "Constraints on low-dimensional string compactifications,"

\footnotetext{
${ }^{10} \mathrm{An}$ anti-D3 brane can dissolve into an anti self-dual flux in a D7 brane, so this class of constructions may be related to the original KKLT construction as a different branch of the anti-D3 configuration space in absence of D5 brane charge. We thank S. Kachru, J. Maldacena, E. Silverstein and A. Uranga for the discussion of this possibility.
} 
Nucl. Phys. B 480 (1996) 213 [arXiv:hep-th/9606122]; K. Dasgupta, G. Rajesh, and S. Sethi, "M theory, orientifolds and G-flux," JHEP 08 (1999) 023. T. Taylor and C. Vafa, "RR flux on Calabi-Yau and partial supersymmetry breaking," Phys. Lett.

B474, 130 (2000). B. R. Greene, K. Schalm, and G. Shiu, "Warped compactifications in M and F theory," Nucl. Phys. B584 (2000) 480-508. C. S. Chan, P. L. Paul, and H. Verlinde, "A note on warped string compactification," Nucl. Phys. B581 (2000) 156-164. K. Becker and M. Becker, "M-Theory on Eight-Manifolds," Nucl. Phys. B477 (1996) 155-167. M. Graña and J. Polchinski, "Supersymmetric three-form flux perturbations on AdS(5)," Phys. Rev. D63 (2001) 026001. S. S. Gubser, "Supersymmetry and F-theory realization of the deformed conifold with three-form flux," hep-th/0010010. S. Kachru, M. Schulz, and S. Trivedi, "Moduli stabilization from fluxes in a simple IIB orientifold," hep-th/0201028. A. R. Frey and J. Polchinski, "N = 3 warped compactifications," Phys. Rev. D65 (2002) 126009. P. K. Tripathy and S. P. Trivedi, "Compactification with flux on K3 and tori," JHEP 03 (2003) 028; S. P. de Alwis, "On potentials from fluxes," hep-th/0307084.

[3] S. B. Giddings, S. Kachru and J. Polchinski, "Hierarchies from fluxes in string compactifications," Phys. Rev. D66, 106006 (2002).

[4] S. Gukov, C. Vafa and E. Witten, "CFTs from Calabi-Yau Fourfolds," Nucl. Phys. B584, 69 (2000).

[5] S. Kachru, J. Pearson and H. Verlinde, "Brane/Flux Annihilation and the String Dual of a Non-Supersymmetric Field Theory," JHEP 0206, 021 (2002) [arXiv:hep-th/0112197].

[6] R. Bousso and J. Polchinski, "Quantization of four-form fluxes and dynamical neutralization of the cosmological constant," JHEP 0006 (2000) 006, arXiv:hep-th/0004134; J. L. Feng, J. March-Russell, S. Sethi and F. Wilczek, Nucl. Phys. B 602 (2001) 307 [arXiv:hep-th/0005276].

[7] W. Y. Chuang, A. Saltman, and E. Silverstein, in progress; http://online.kitp.ucsb.edu/online/strings03/silverstein/.

[8] E. Cremmer, S. Ferrara, C. Kounnas and D.V. Nanonpoulos, "Naturally vanishing cosmological constant in $N=1$ supergravity," Phys. Lett. B133, 61 (1983); J. Ellis, A.B. Lahanas, D.V. Nanopoulos and K. Tamvakis, "No-scale Supersymmetric Standard Model," Phys. Lett. B134, 429 (1984).

[9] J.- P. Derendinger, L. E. Ibáñez and H. P. Nilles, "On The Low-Energy D = 4, N=1 Supergravity Theory Extracted From The D = 10, N=1 Superstring," Phys. Lett. B 
155 (1985) 65; M. Dine, R. Rohm, N. Seiberg and E. Witten, "Gluino Condensation In Superstring Models," Phys. Lett. B 156 (1985) 55; C.P. Burgess, J.-P. Derendinger, F. Quevedo and M. Quirós, "On Gaugino Condensation with Field-Dependent Gauge Couplings" Annals of Physics, 250 (1996) 193-233.

[10] C. Escoda, M. Gómez-Reino and F. Quevedo, "Saltatory de Sitter string vacua," arXiv:hep-th/0307160.

[11] N. V. Krasnikov, "On Supersymmetry Breaking In Superstring Theories," Phys. Lett. B 193 (1987) 37.

[12] S. Kachru, R. Kallosh, A. Linde, J. Maldacena, L. McAllister and S. P. Trivedi, "Towards inflation in string theory," arXiv:hep-th/0308055.

[13] I. Brunner, M. R. Douglas, A. E. Lawrence and C. Romelsberger, "D-branes on the quintic," JHEP 0008 (2000) 015 [arXiv:hep-th/9906200]; E. Witten, "BPS bound states of D0-D6 and D0-D8 systems in a B-field," JHEP 0204 (2002) 012 [arXiv:hep-th/0012054]; S. Kachru and J. McGreevy, "Supersymmetric three-cycles and (super)symmetry breaking," Phys. Rev. D 61 (2000) 026001 [arXiv:hep-th/9908135]; R. Blumenhagen, D. Lüst and T. R. Taylor, "Moduli stabilization in chiral type IIB orientifold models with fluxes," Nucl. Phys. B 663 (2003) 319 [arXiv:hep-th/0303016]; J. F. Cascales and A. M. Uranga, "Chiral 4d N = 1 string vacua with D-branes and NSNS and RR fluxes," JHEP 0305 (2003) 011 [arXiv:hep-th/0303024].

[14] For T-dual versions see for instance: R. Blumenhagen, L. Goerlich, B. Kors and D. Lust, "Noncommutative compactifications of type I strings on tori with magnetic background flux," JHEP 0010 (2000) 006 [arXiv:hep-th/0007024]; G. Aldazabal, S. Franco, L. E. Ibanez, R. Rabadan and A. M. Uranga, "Intersecting brane worlds," JHEP 0102 (2001) 047 [arXiv:hep-ph/0011132]; M. Cvetic, G. Shiu and

A. M. Uranga, "Chiral four-dimensional $\mathrm{N}=1$ supersymmetric type IIA orientifolds from intersecting D6-branes," Nucl. Phys. B 615 (2001) 3 [arXiv:hep-th/0107166]; D. Cremades, L. E. Ibanez and F. Marchesano, "Intersecting brane models of particle physics and the Higgs mechanism," JHEP 0207 (2002) 022 [arXiv:hep-th/0203160]; "SUSY quivers, intersecting branes and the modest hierarchy problem," JHEP 0207 (2002) 009 [arXiv:hep-th/0201205].

[15] C. Herdeiro, S. Hirano and R. Kallosh, "String theory and hybrid inflation / acceleration," JHEP 0112, 027 (2001) [arXiv:hep-th/0110271]; K. Dasgupta, 
C. Herdeiro, S. Hirano and R. Kallosh, "D3/D7 inflationary model and M-theory," Phys. Rev. D 65, 126002 (2002) [arXiv:hep-th/0203019].

[16] P. Binetruy and E. Dudas, "Gaugino condensation and the anomalous U(1)," Phys. Lett. B 389 (1996) 503 [arXiv:hep-th/9607172]; G. R. Dvali and A. Pomarol, "Anomalous U(1) as a mediator of supersymmetry breaking," Phys. Rev. Lett. 77 (1996) 3728 [arXiv:hep-ph/9607383].

[17] P. Binetruy and G. R. Dvali, "D-term inflation," Phys. Lett. B 388, 241 (1996) [arXiv:hep-ph/9606342]; E. Halyo, "Hybrid inflation from supergravity D-terms," Phys. Lett. B 387, 43 (1996) [arXiv:hep-ph/9606423];

[18] J. A. Casas, J. M. Moreno, C. Muñoz and M. Quirós, "Cosmological Implications Of An Anomalous U(1): Inflation, Cosmic Strings And Constraints On Superstring Parameters," Nucl. Phys. B 328 (1989) 272

[19] K. Becker, M. Becker, K. Dasgupta and S. Prokushkin, "Properties of heterotic vacua from superpotentials," Nucl. Phys. B 666 (2003) 144 [arXiv:hep-th/0304001]; S. Gukov, S. Kachru, X. Liu and L. McAllister, "Heterotic Moduli Stabilization with Fractional Chern-Simons Invariants," (to appear); K. Becker, M. Becker, K. Dasgupta, P. S. Green and E. Sharpe, "Compactifications of Heterotic Strings on Non-Kahler Complex Manifolds-II," (to appear).

[20] E. Witten, "Dimensional Reduction Of Superstring Models," Phys. Lett. B 155 (1985) 151; C. P. Burgess, A. Font and F. Quevedo, "Low-Energy Effective Action For The Superstring," Nucl. Phys. B 272 (1986) 661.

[21] A. Font, L. E. Ibánez, D. Lüst and F. Quevedo, "Supersymmetry Breaking From Duality Invariant Gaugino Condensation," Phys. Lett. B 245 (1990) 401; S. Ferrara, N. Magnoli, T. R. Taylor and G. Veneziano, "Duality And Supersymmetry Breaking In String Theory," Phys. Lett. B 245 (1990) 409.

[22] M. Cvetič, A. Font, L. E. Ibáñez, D. Lüst and F. Quevedo, "Target space duality, supersymmetry breaking and the stability of classical string vacua," Nucl. Phys. B 361 (1991) 194.

[23] B. de Carlos, J. A. Casas and C. Muñoz, "Supersymmetry breaking and determination of the unification gauge coupling constant in string theories," Nucl. Phys. B 399 (1993) 623 [arXiv:hep-th/9204012].

[24] M. Dine, N. Seiberg and E. Witten, "Fayet-Iliopoulos Terms In String Theory," Nucl. Phys. B 289 (1987) 589. 
[25] N. Seiberg and E. Witten, "String theory and noncommutative geometry," JHEP 9909, 032 (1999) [arXiv:hep-th/9908142].

[26] G. R. Dvali and S. H. Tye, "Brane inflation," Phys. Lett. B 450 (1999) 72 [arXiv:hep-ph/9812483]; C. P. Burgess, M. Majumdar, D. Nolte, F. Quevedo, G. Rajesh and R. J. Zhang, "The inflationary brane-antibrane universe," JHEP 0107 (2001) 047 [arXiv:hep-th/0105204]; G. R. Dvali, Q. Shafi and S. Solganik, "D-brane inflation," arXiv:hep-th/0105203; J. Garcia-Bellido, R. Rabadán and F. Zamora, "Inflationary scenarios from branes at angles," JHEP 0201 (2002) 036 [arXiv:hep-th/0112147]; C. P. Burgess, P. Martineau, F. Quevedo, G. Rajesh and R. J. Zhang, "Brane antibrane inflation in orbifold and orientifold models," JHEP 0203 (2002) 052 [arXiv:hep-th/0111025]; N. Jones, H. Stoica and S. H. Tye, JHEP 0207 (2002) 051 [arXiv:hep-th/0203163]; M. Gomez-Reino and I. Zavala, "Recombination of intersecting D-branes and cosmological inflation," JHEP 0209 (2002) 020 [arXiv:hep-th/0207278]. 